\title{
HOST PLANTS AND DISTRIBUTION OF SOME WHITEFLIES SPECIES (HEMIPTERA, ALEYRODIDAE) IN THE MIDDLE OF IRAQ*
}

\author{
I. M. Al-Mallo** and M.S. Abdul-Rassoul*** \\ **Department of Plant Protection, College of Agriculture, University of \\ Baghdad, Baghdad, Iraq \\ *** Iraq Natural History Research Center and Museum, University of \\ Baghdad, Baghdad, Iraq \\ ****Corresponding author: msabr_1942@yahoo.com
}

\begin{abstract}
Ten species of whiteflies (Hemiptera, Aleyrodidae) representing six genera were studied from a collection from different localities in the middle of Iraq. These species are Acaudaleyrodes rachipora (Singh, 1931); Bemisia afer (Priesner and Hosny,1934); Bemisia tabaci (Gennadius, 1889); Dialeurodes citri (Ashmead,1885); Dialeurodes kirkaldy (Kotinsky, 1907); Neomaskellia andropogonis Corbett, 1926; Siphoninus phillyreae (Haliday, 1835); Trialeurodes ricini (Misra, 1924); Trialeurodes vapovariorum (Westwood,1856) and Trialeurodes irakeensis (Al-Malo and Abdul-Rassoul, 2000). Notes are given on their localities, date of collection and host plants.
\end{abstract}

Key words: Aleyrodidae, Distribution, Host plants, Iraq, Whiteflies.

\section{INTRODUCTION}

Whiteflies are small insects belonging to the family Aleyrodidae order Hemiptera; it comprised 1556 species in 161 genera from all over the world (Evans , 2007); they feed on about 164 plant families (Mound and Halsey, 1978 ). Duffus and Flock (1982) indicated that as a result of their feeding they transmit about thirty viral diseases, in addition to this the secretion of the honeydew causes fungi growth.

At that time, there was no taxonomic study for this family in Iraq, but there were recorded some species such as Hussain (1963) who reported Bemisia tabaci (Gennadius,1889); followed by Derwesh (1965) who added two more species Acaudaleyrodes rachipora (Singh, 1931) (= Acaudaleyrodes citti (Priesner and Hosny, 1934)) and Trialeurodes sp.; two further species were reported by Mound and Halsey (1978) namely Trialeurodes rara Singh, (1931) and Trialeurodes ricini (Misra, 1924). Subsequently, one species was added by Swailm et al. (1974) namely Siphoninus granati (Priesner and Hosny, 1934), which was a synonym of $S$. phillyreae (Mound and Halsey, 1978) and five spices were added to the Iraqi fauna by Farag and Amine (1982). These were Aleyrodes proletella (Linnaeus, 1758), Bemisia afer (Priesner and Hosny, 1934), Bemisia hanocki (group), Siphoninus phillyreae (Haliday,1835), and Trialeurodes porosus (Priesner and Hosny, 1934).

*This paper is part of M.Sc. thesis by the first author. 
Host Plants and Distribution of Some Whiteflies

It appeared later that one of these species T. porosus was a synonym of Aleuroclava porosus (Priesner and Hosny, 1937) (Evans, 2007), Al-Malo and Abdul-Rassoul (2000) described Trialeurodes irakeensis as a new species to the science.

\section{MATERIALS AND METHODS}

Pupal cases of whitefly have been collected from infected plant leaves during the years 1986 to 1988 , in the central region of Iraq; the specimens were collected from about 50 species of host plants belonging to 25 plant families, and kept in the petri dishes. The infected leaves by the whitefly were examined by a binocular dissecting microscope to distinguish the pupal case with a fine needle, pupal cases were mounted on slides according to Bink (1979), and examined under a compound microscope by using an ocular micrometer to measure length of the insect. Identification keys of Mound (1965, 1966), Habib and Farag (1970) and Sampson and Drews (1956) were used to identify species collected during this study.

\section{List of species reported in this study:}

\section{RESULTS AND DISCUSSION}

Acaudaleyrodes rachipora (Singh,1931) $(=A$. citri Priesner and Hosny, 1934)

Materials Examined: 12 pupal case, Baghdad 21.II.1987, 26. V.1987, 9.IV.1988 on leaves of Zizyphus spina-christi (Linne.) Wild. (Rhamnaceae); 4 pupal case, Karbala, 10.III.1988, 1.IV.1988 on leaves of Zizyphus spina-christi (Linne.) Wild. (Rhamnaceae); 2 pupal case, Baghdad, 27.XII.1987 on leaves of Punica granatum Linne. (Lythraceae); 3 pupal case, Diyala, 3.II.1988 on leaves of Punica granatum Linne. (Lythraceae).

\section{Bemisia afer (Priesner and Hosny, 1934)}

Materials Examined: 16 pupal case, Baghdad, 4,10.Vii.1988 on leaves of Rosa sp. (Rosaceae); 6 pupal cases, Baghdad, 9, 25.IV.1988, 26.v.1988 on leaves of Zizyphus spinachristi (Linne.) Wild. (Rhamnaceae); 3pupal case, Karbala, 14.VI.1988 on leaves of Zizyphus spina-christi (Linne.) Wild. (Rhamnaceae); 6 pupal case, Baghdad, 23, 27.IV.,1.V., 14.VII.1988 on leaves of Citrus sp. (Rutaceae); 4 pupal case, Diyala, 4.V, 4.VII., 16.VI.1988 on leaves of Citrus sp. (Rutaceae); 2 pupal case, Baghdad, 27.IV.1988 on leaves of Vitis vinifera Linne.(Vitaceae); 1 pupal cases, Baghdad, 7.VI.1988 on leaves of Punica granatum Linne. var. nana (Punicaceae); 1 pupal case, Baghdad, 18.VI.1988 on leaves of Lagerstroemia indica Linne.( Lythraceae).

\section{Bemisia tabaci (Gennadius, 1889)}

Materials Examined: 3 pupal case,Baghdad, 21.VIII.1987 on Hibiscus esculentus L. (Malvaceae); 10 pupal case,Baghdad, 20.VIII.1978, 22. VI.1988 on Cucumis sativus L. (Cucurbitaceae); 3 pupal case,Baghdad, 10.VII.1987 on Gossypium hirsutum L. (Malvaceae); 8 pupal case,Baghdad, 1.IX., 22.Xii.1987, 14.IV.1988 on Hibiscus rosa- sinensis L. (Malvaceae) ; 9 pupal case,Baghdad, 20.V.1987, 2, 4.IV1988 on Zinnia elegans Jacq (Asteraceae); 5 pupal case,Baghdad, 30.VI.1988 on Cynanchum acutum L. (Ascclepiadaceae); 10 pupal case, Baghdad, 20.VIII.1987, 18.IX.1988 on Helianthus annuus L. (Asteraceae); 4 pupl case, Baghdad,9, 10, 13.IV. 1988 on Zizyphus spina-christi (Linne.) Wild. (Rhamnaceae); 2 pupl case, Baghdad, 30.VI. 1988 on Prunus armeniaca L. (Rosaceae); 3 pupl case, Baghdad, 7.V.1988 on Capparis spinosa L. (Capparaceae); 2 pupl case, Baghdad, 10.VII.1988, on Verbena hybrid V. (Verbenaceae); 3 pupal case, Baghdad, 21.VIII.1987, on Ricinus communis Linne. (Euphorbiaceae); 5 pupl case, Baghdad, 20.IX.1987 on Sonchus communis L. (Asteraceae); ${ }^{\top}$ pupl case, Baghdad, 26.VI.1988 on Cardaria draba (L). Desv. (Brassicaceae); $r$ pupl case, Baghdad, 18.VI.1988 on Plantago lanceolata L. (Plantaginaceae); 5 pupal case Baghdad, 28.Vi.1988 on Convolvuus arvensis L. 


\section{M. Al-Mallo and M.S. Abdul-Rassoul}

(Convolvulaceae); 5 pupal case Baghdad, 21.VIII.1988 on Solanum melongena L. (Solanaceae); 7 pupal case Baghdad, 21.VIII.1988 on Solanum tuberosum L. (Solanaceae); 5 pupal case Baghdad, 30.VIII.1988 on Capsicum grossum L. (Solanaceae); 3 pupal case Baghdad, 30.VI.1988 on Citrus sp. (Rutaceae); 5 pupal case Baghdad, 20.VII.1987 on Amaranthus atropurpureus Roxb. (Amaranthaceae); 5 pupal case Baghdad, 18.IV.1988 on Rosa sp. (Rosaceae); 4 pupal case Baghdad, 20.VII.1987 on Phanera purpurea (L.) Benth. (Fabaceae); 2 pupal case , Baghdad, 15.V.1987 on Punica granatum var. nana (Lythraceae); 3 pupal case , Baghdad, 16.VI .1988 on Bougainvillea sp. (Nyctaginaceae); 3 pupal case , Baghdad, 16.VI.1988 on Bougainvillea sp. (Nyctaginaceae); 2 pupal case , Baghdad, 27.IV .1988 on Ficus carica L. (Moraceae); 6 pupal case , Baghdad, 3.X .1988 on Nicotiana tabacum L. (Solanaceae); 5 pupal case , Hila, 8.X .1987 on Hibiscus esculentus L. (Malvaceae); 6 pupal case , Anbar, 21.VIII .1987 on Chrozophora tinctoria (L.) Juss. ( = Chrozophora varbascifolia (Wild.) Juss. (Euphorbiaceae); 4 pupal case , Anbar,10.X.1987 on Tagetes sp. (Asteraceae); 4 pupal case , Anbar,10.X.1987 on Solanum melongena L. (Solanaceae); 4 pupal case , Anbar,15.X.1987 on Ipomoea batatas (L.) Poir (Convolvolaceae); 4 pupal case , Anbar,3.XI.1987 on Nicotiana tabacum L. (Solanaceae); 7 pupal case , Anbar,10.X.1987 on Helianthus tuberosus L. (Asteraceae); 3 pupal case , Diyalar,10.X.1987 on Solanum nigrum L. (Solanaceae); 4 pupal case , Diyala,3.X.1987 on Chrozophora tinctoria (L.) Juss. (Euphorbiaceae); 5 pupal case , Diyalar,3.X.1987 on Euphorbia helioscopia L. (Euphorbiaceae); 3 pupal case , Diyala,3.X.1987 on Zinnia elegans Jacq. (Asteraceae); 3 pupal case , Wasit, 3.VI., 4.VII.1988 on Zinnia elegans Jacq. (Asteraceae); 7 pupal case, Wasit, 29.VI.,20.VII., 23.VII.1987 on Hibiscus rosa - sinensis L. (Malvaceae); 7 pupal case , Karbala, 20.VII.1987 on Brassica napus L. (Brassicaceae); 3 pupal case, Karbala, 20.VII.1987 on Helianthus annuus L. (Asteraceae).

\section{Dialeurodes citri (Ashmead, 1885)}

Materials Examined: 7 pupal case, Baghdad, 17.IV. 1988, 4.V.1988 on leaves of Jasminum sambac (L.) Aiton . (Oleaceae).

\section{Dialeurodes kirkaldyi (Kotinsk, 1907)}

Materials Examined: 11 pupal case, Baghdad, 17, 19.IV., 14.VII.1988 on leaves of Jasminum sambac (L.) Aiton. (Oleaceae).

Neomaskellia andropogonis Corbett, 1926

Materials Examined: 14 pupal case, Anbar, 30.X.1987 on Imperata cylindrica (Linne.) P.Beauv (Poaceae).

\section{Siphoninus phillyreae (Halliday, 1835)}

Materials Examined: 13 pupal case, Baghdad, 20.VIII.1987, 24.IV, 5.VI., 4.VII.1988 on Pyrus communis Linne. (Rosaceae); 8 pupal case, Baghdad, 12.VI., 10.VII.1988 on Punica granatum Linne. (Lythraceae); 10 pupal case, Anbar, 7.VI.1988 on Punica granatum Linne. (Lythraceae); Karbala, 24.V.1987 on Citrus sp. (Rutaceae); 12 pupal case Diyala, 12.V.1987 on Citrus sp. (Rutaceae).

\section{Trialeurodes ricini (Misra, 1924) (=T. rara Signh, 1931)}

Materials Examined:43 pupal case, Baghdad, 4, 9.IV.1988 on Zizyphus spina-christi (Linne.) Wild. (Rhamnaceae); 21 pupal case, Baghdad, 22.XII.1986, 24.III,1987, 4.V.1988 on Citrus sp. (Rutaceae); 4 pupal case, Baghdad, 25.III., 4.IV.1988 on Rosa sp. (Rosaceae); 7 pupal case, 25.III.1987, 72 pupal case, Baghdad, 25.X.1987 on Ricinus communis Linne. (Euphorbiaceae); 7 pupal case, Baghdad, 6.IV. 1988, 14.VII.1988 on Rosa sp. (Rosaceae); 3 pupal case, Baghdad, 28.VI.1988 on Hibiscus rosa-sinensis Linne. (Malvaceae); 3 pupal case, 
Host Plants and Distribution of Some Whiteflies

Baghdad, 29.X.1987 on Celosia cristata Linne. (Amarantaceae); 5 pupal case, 25.I.1987, 6 pupal case, 4, 24.V.1987 Karbala on Citrus sp. (Rutaceae); 2 pupal case, Karbala, 3.X.1987 on Ricinus communis Linne. (Euphorbiaceae); Babil , 20.XII.1986, 4.VII.1988, 8.V.1988 on Citrus sp. (Rutaceae); 2 pupal case, Wasit, 23.V.1987on Citrus sp. (Rutaceae); 5 pupal case, Diyala, 3, 4.IV.1988 on Rosa sp. (Rosaceae); 3 pupal case, Anbar, 8.III.1988 on Rosa sp. (Rosaceae).

\section{Trialeurodes vaporariorum (Westwood, 1856)}

Materials Examined: Mounting (Rutaceae); 3 pupal case, Diyala, 17.IV.1987 on Citrus sp. (Rutaceae).

\section{Trialeurodes irakeensis Al-Malo and Abdul-Rassoul, 2000}

Materials Examined: 4 pupal case, Baghdad, 3.IV.1987 on Hibiscus rosa-sinensis Linne.

(Malvaceae).

\section{LITERETURE CITED}

Al-Malo, I.M. and Abdul-Rasoul, M.S. 2000. A new species of genus Trialeurodes Cockere from Iraq (Homoptera, Aleyrodidae). Bulletin of the Iraq Natural History Museum, 9(2): $19-23$

Bink, F. A. 1979. Methods for mounting Aleyrodidae specimens. Entomologische berichtea (Amsterdam), 39(10): 158-160.

Derwesh, A. I. 1965 . A preliminary list of identified insects and some arachnids of Iraq . Directorate General of Agriculture Research and Projects , Baghdad , Bulletin $121 \mathrm{pp}$.

Duffus, J.E. and Flock, R.A. 1982. Whitefly -transmitted disease complex of the desert southwest, California. Agriculture, 36: 4-6.

Evans, G.A. 2007. The whiteflies (Hemiptera: Aleyrodidae) of the world and their host plants and natural enemies, 708pp.

Farag, A.F. and Amin, A.H. 1982. New record of six species of whiteflies in Iraq, with numerical density study for the cotton whitefly Bemisia tabaci (Genn.) on economically important crops. Mesopotamia Journal of Agriculture, 16 (1): 93104. (In Arabic).

Habib, A.L. and Farag, F.A. 1970. Studies on nine common Aleurodids of Egypt. Bulletin of the Entomological Society of Egypt, 54: 1-41.

Hussain, A . A . 1963. Provisional list of Insect pests and Bibliography of Insect fauna of Iraq. Bulletin of the College of Science, Baghdad, $7: 43-83$.

Mound, L.A. 1965. An introduction to the Aleyrodidae of western Africa (Homoptera). Bulletin of the British Museum of Natural History, 17(3): 113-160.

Mound, L.A. 1966. A revision of the British Aleyrodidae (Hemiptera: Homoptera). Bulletin of the British Museum of Natural History, 17(9): 397- 428. 


\section{M. Al-Mallo and M.S. Abdul-Rassoul}

Mound, L.A. and Halsey, S.H. 1978. White fly of the world. British Museum and John Wiley and Sons, $340 \mathrm{pp}$.

Sampson, W.W. and Drews, E.A. 1956. Keys to the genera of the Aleyrodidae and notes on certain genera (Homoptera: Aleyrodidae). Annals and Magazine of Natural History, series 12, 9: 689-697. 
Bull. Iraq nat. Hist. Mus.

(2017) 14 (4): 295-300

$$
\begin{aligned}
& \text { المضايف النباتية و الانتشار لبعض أنو اع الذباب الابيض } \\
& \text { (Hemiptera, Aleyrodidae) } \\
& \text { في وسط العراق } \\
& \text { إيمان محمد المالو* * و محمد صالح عبد الرسول } \\
& \text { *قسم وقاية النبات، كلية الزر اعة، جامعة بغداد } \\
& \text { مركز بحوث ورتحف التاريخ الطبيعي، جامعة بغداد لزية بغاد }
\end{aligned}
$$

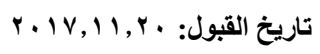

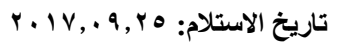

\section{الخلاصة}

سجلت عشرة انواع من الذباب الابيض (رتبة نصفية الاجنحة: عائلة الذباب الابيض)

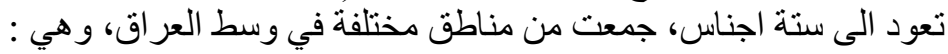

Acaudaleyrodes rachipora (Singh, 1931); Bemisia tabaci (Gennadius,1889); Bemisia afer (Priesner and Hosny, 1934); Dialeurodes kirkaldy (Kotinsky, 1907); Dialeurodes citri (Ashmead,1885); Siphoninus phillyreae (Haliday,1835); Neomaskellia andropogonis Corbett, 1926; Trialeurodes vapovariorum (Westwood) 1856; Trialeurodes ricini (Misra,1924) Trialeurodes irakeensis AlMalo and Abdul-Rassoul ,2000.

كما سجلت مناطق التوزيع، وتاريخ الجمع و المضايف النباتية لكل نوع منها. 